charged electrons moving through a lattice of positive ions whereas, in the case of ${ }^{3} \mathrm{He}$, there is no lattice and the fermions are neutral atoms. It is not obvious, therefore, just how far the analogy can be taken.

\section{SOLID STATE \\ Laser Writing}

from a Correspondent

THE past four or five years have seen a great increase in research on amorphous semiconductors. This has followed a rather longer period devoted to crystalline materials and the development of very sophisticated semiconductor devices. Amorphous materials have been investigated principally in the form of glasses, and the particular property that has, perhaps, stimulated the most interest is their electrical behaviour. Devices made from such materials show either memory or threshold switching depending on their composition. (Memory switching is the phenomenon by which the material may exhibit either a high or a low resistance state.)

It is now fairly certain that memory switching is associated with the transition from amorphicity to a crystalline state, in the form of thin filaments between the electrodes caused by electrical heating followed by slow cooling. The reverse process involves heating followed by a rapid cooling or quenching to allow the material to devitrify or revert to the amorphous state.

It is now possible to induce a similar condition in amorphous materials by the interaction with light from lasers. Rapid crystallization and equally rapid devitrification of amorphous chalcogenides (for example $\mathrm{Te}_{81} \mathrm{Ge}_{15} \mathrm{Sb}_{2} \mathrm{~S}_{2}$ ) have been observed when they are exposed to short laser pulses (Appl. Phys. Lett., $18,254 ; 1971)$. This process of optical switching is made evident by the sharp change in the optical transmission and reflexion properties of those areas so exposed. It was suggested that the physical change is attributable to a bondweakening mechanism or photocrystallization process, but a more recent paper (Appl. Phys. Lett., 22, 48 ; 1973) gives evidence that in a similar glass the initial devitrification is temperature activated. This paper also shows how by using the same laser it is possible to produce a crystalline area (memory state), to measure the change in optical transmission (read out), and to revert the same area to the amorphous state (erase).

These optical memories can also be operated in the so-called "reverse mode" (J. Appl. Phys., 43, 4688 ; 1972). An initial uniform crystalline state is obtained by heating the glass thin film to $90^{\circ} \mathrm{C}$. The interaction with laser light produces an amorphous pattern which can be first "read" and then "erased" by heating once more to $90^{\circ} \mathrm{C}$ and cooling slowly. The advantages of this reverse mode of operation are that it gives a much faster "write time", allowing the rather slower reverse process to be used for "erasing".

It is very difficult at this stage of their development to predict the future for these devices, but undoubtedly they will be the subject of many more investigations, if only to resolve further the mechanisms involved in this phenomenon.

\section{SAN ANDREAS FAULT Pore Pressure and Creep}

CREEP occurring in the central part of the San Andreas Fault-a right-lateral strike-slip fault running through California-can be related to changes in the pore pressure of water, as measured in a well $150 \mathrm{~m}$ deep, near Hollister. In this part of the fault the total creep of $1.2 \mathrm{~cm} \mathrm{yr}^{-1}$ occurs as a series of small creep events.

Johnson, Kovach, Nur and Booker report (J. Geophys. Res., 78, 851 ; 1973) that both the maximum pore pressure and the offset in water level in the well are linearly related to the total creep in motions which occur within hours of anomalous changes in water level.

Three creep events, of magnitudes $4 \mathrm{~mm}, 3 \mathrm{~mm}$ and $2 \mathrm{~mm}$, were associated with changes in water level of $+5.6 \mathrm{~cm}$, $-4.1 \mathrm{~cm}$ and $+3 \mathrm{~cm}$, respectively. The water level started to change $4 \mathrm{~h}$ before the first event, $1.5 \mathrm{~h}$ after the second and $8 \mathrm{~h}$ before the third. The recorder used is sensitive only to within $\pm 2 \mathrm{~mm}$, insufficient for changes caused by Earth tides, for example, to have been monitored. The one to one correspondence - all anomalous water level changes were associated with fault movement-is particularly striking, and follows several recent experiments, notably in Colorado, where earthquakes have been produced by deliberate injection of water into the pores.

The success of those experiments has led to speculation that it might be possible to make the San Andreas Fault safe by drilling deep wells along the fault line and "locking" it by pumping water out. Selected regions of the fault could then be isolated and water pumped in to liberate the slip. Repeated small man-made earthquakes could ensure a relatively smooth slip without the sudden massive jerks which make the region so dangerous at present-or so the argument runs.

Johnson et al. point out that it is not possible, as yet, to say whether the transient pore pressure is produced by creep or the creep by changes in fluid levels. But deeper wells might be able to detect strains and pressure changes deeper in the Earth, monitoring pore pressure changes at different distances from the active region of the fault. If such monitoring proves effective, it may become possible to predict some of the behaviour of the active fault, or to understand the delicate balance of pore pressure and fault activity sufficiently well to permit experiments aimed at stabilizing the fault, by adding or removing water as required. That, however, is certainly a very long term prospect indeed.

\title{
Doppler Measurements of Metal Flow Velocilies
}

Ultrasonic Doppler velocimeters have been found useful in the measurement of fluid flow, for example blood flow. The technique depends, of course, on the presence of inhomogeneities in the fluid, from which the sound waves can be reflected. This would seem to make the technique unsuitable for studying liquid metal flow, but in the best traditions of "suck it and see" Fowlis has attempted to use such a velocimeter to measure the flow of both mercury and the liquid alloy $\mathrm{NaK}$ (47 per cent $\mathrm{Na}$ by weight, 53 per cent $K$ ). To his own surprise, he obtained a strong Doppler signal for both metals (see next Monday's Nature Physical Science, March 5).

The photograph shows the apparatus used, in which the Doppler shift of ultrasonic waves backscattered from the moving metal is recorded. The metal flows under gravity between two reservoirs along a tube in which the ultra- sonic probe is mounted. The Doppler shift frequency range can be directly examined with the analyser and oscilloscope to the left.

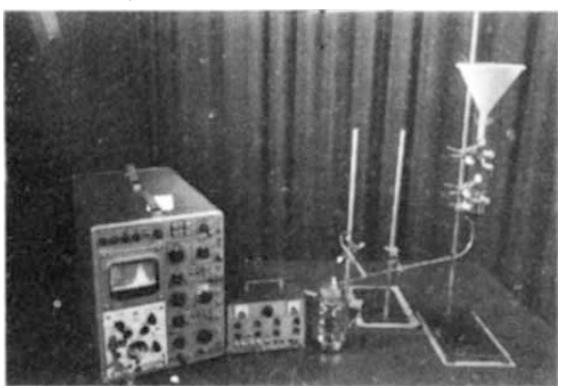

The source of the backscatter is unknown, although Fowlis does point to dust particles or microscopic bubbles as possible candidates; the important practical point, however, is that the system offers a cheap and apparently accurate means of measuring such flow, no matter how the effect works. 